\title{
Bibliographie
}

\section{Sources primaires}

Albert le Grand, Saint, Le Monde minéral: les pierres. De mineralibus (livres I et II). Présentation, traduction et commentaires par Michel Angel, Sagesses chrétiennes (Paris: Les Éditions du cerf, 1995)

Aneau, Barthélemy, Alector ou le coq: Histoire fabuleuse, éd. Marie Madeleine Fontaine, 2 tomes, Textes Littéraires Français, 469 (Genève: Droz, 1996)

Apollonius de Rhodes, Apollonius Rhodius: Argonautica, éd. et trad. R.C. Seaton, Loeb Classical Library (Cambridge, MA: Harvard University Press; Londres: Heinemann, 1967)

Arena, Antonius, Ad suos compagnones... 1531, édition bilingue; texte établi, traduit, annoté et commenté par Marie-Joëlle Louison-Lassablière (Paris: Champion, 2012)

- Ad suos compagnones studiantes (Poitiers: Jacques Bouchet, 1546)

- Ad suos Compagniones Studiantes, s.l., 1529 (BL 1070.b.2)

Baïf, Jean-Antoine de, Mimes, Enseignemens et Proverbes, éd. Jean Vignes, Textes Littéraires Français 411 (Genève: Droz, 1992)

- Euvres en rime de lan Antoine de Baïf, éd. Charles Marty-Laveaux, La Pléiade Françoise (Genève: Slatkine Reprints, s.d.)

Baudelaire, Charles, Les Fleurs du Mal, éd. Antoine Adam, Classiques Garnier (Paris: Garnier, 1961) 
Belleau, Remy, Euvres poétiques, édition critique sous la direction de Guy Demerson (Paris: Champion, 1995-2003)

- La Reconnue, éd. Jean Braybrook (Genève: Droz, 1989)

- Commentaire au Second Livre des Amours de Ronsard, publié par M.M. Fontaine et François Lecercle (Genève: Droz, 1986)

- Les Amours et nouveaux eschanges des pierres précieuses, éd. Maurice F. Verdier (Genève: Droz; Paris: Minard, 1973)

- Fuvres poetiques de Remy Belleav. Avec une Notice biographique et des notes, éd. Ch. Marty-Laveaux, 2 vols, La Pléiade Françoise (Paris: Lemerre, 1878; Genève: Slatkine, 1965)

- La Bergerie. Texte de l'édition de 1565, éd. Doris Delacourcelle (Genève: Droz et Lille: Giard, 1954)

- La Première Jovrnée de La Bergerie de Remy Belleav (Paris: Société des Médecins bibliophiles, 1945)

- Jan qui ne peult [Suivi de: Sur une bouche], éd. René-Louis Doyon (Paris: Robert Coulouma, 1929). BnF: Rés.p.Ye.1981

- Les Amours et nouveaux eschanges des pierres precieuses de Remy Belleau, suivis d'autres poésies du même auteur, éd. Adolphe Van Bever, La Pléiade française (Paris: Sansot, 1909)

- Fuvres complètes de Remy Belleau, éd. A. Gouverneur, 3 vols (Paris: A. Franck; Nogent-le-Rotrou: A. Gouverneur, 1867)

- L'Eschole de Salerne en vers Burlesques. Et poema macaronicvm de bello Hvgvenotico, éd. Louis Martin, MD (Rouen: Clement Malassis, 1660). Bodleian, Oxford; voir p. 81-100 et, pour une traduction, p. 101-144

- Les CFvrres poetiqves de Remy Belleav. Redigees en deux tomes. Reueuës \& corrigees en ceste derniere impression (Rouen: Claude le Villain, 1604; Rouen: Thomas Daré, 1604; Rouen: Jean Berthelin, 1604)

- La Reconnve, comedie (Rouen: Thomas Daré, 1604) 
- Les CEvvres poetiqves de Remy Belleav. Redigees en deux tomes. Reueuës $\&$ corrigees en ceste derniere impression (Paris: Gilles Gilles, 1585; Paris: Mamert Patisson, 1585)

- Les CEvres poetiqves de Remy Belleav. Redigees en deux tomes (Paris: Mamert Patisson, 1578)

- Remigij Bellaquei Poetce Tvmvlvs (Paris: Mamert Patisson, 1577)

- Les Amours et novveavx eschanges des pierres precieuses [...] Discours de la vanité [...] Eclogues sacrees (Paris: Mamert Patisson, 1576)

- La Bergerie de R. Belleav, divisee en vne premiere \& seconde lournee (Paris: Gilles Gilles, 1572)

- Larmes svr le Trespas de Monseigneur René de Lorraine, et de Madame Lovyse de rieux Marquis \& Marquise d'Elbeuf. Ensemble le Tombeav de Monseigneur François de Lorraine Duc de Guyse \& Pair de France. Par R. Belleav (Paris: Gabriel Buon, 1566; il s'agit d'une édition mal soignée, avec beaucoup de coquilles)

- La Bergerie (Paris: Gilles Gilles, 1565)

- Chant Pastoral svr la mort de loachim Dv Bellay Angevin. Par Remi Belleav (Paris: Robert Estienne, 1560)

- Chant Pastoral de la Paix. Par R. Belleav (Paris: André Wechel, 1559)

- Ode presentée à monseigneur le Duc de Guyse à son retour de Calais (Paris: André Wechel, 1558). Wren Library, Trinity College, Cambridge, M.13.485

Belleforest, François de, La Cosmographie universelle de tout le monde (Paris: M. Sonnius, 1575)

Besson, Jacques, Le Cosmolabe, ou Instrument Universel, concernant Toutes Observations qui se peuvent faire Par les Sciences Mathematiques, Tant au Ciel, en la Terre, comme en la Mer (Paris: Ph. G. de Roville, 1567)

Bèze, Théodore de, Du Droit des magistrats sur leurs sujets (1574), éd. R.M. Kingdon (Genève: Droz, 1971)

- Les Juvenilia de Théodore de Bèze, éd. Alexandre Machard (Paris: Isidore Liseux, 1879) 
Bible, La Bible de Poche Jérusalem (s.l.: Desclée de Brouwer, 1970)

Bloomfield, William, The Compendiary of the noble science of alchemy compiled by Mr Willm Blomefeild philosopher \& bacheler of physick admitted by king Henry the $8^{\text {th }}$ of most famous memory. Anno Domini 1557. Voir http://www.levity.com/ alchemy/bloomfld.html

Boaistuau, Pierre, Histoires prodigieuses, éd. Gisèle Mathieu-Castellani, Fleuron, 87 (Paris-Genève: Slatkine, 1996)

Calvin, Jean, Articles, éd. Jacques de Senarclens (Genève: s.n., 1941)

- loannis Calvini opera quae supersunt omnia (Corpus reformatorum), éd. Guilielmus Baum, Eduardus Cunitz et Eduardus Reuss, 59 vols (Brunswick: Schwetschke (Bruhn), 1868)

Camus, Albert, L'Étranger, éd. Ray Davison, Routledge Foreign Literature Classics (Londres et New York: Routledge, 2007; première édition Routledge 1988)

Cardan, Jérôme (Cardano, Girolamo), Les Livres de Hierome Cardanus [...] intitulés de la subtilité [...] traduis de latin en françois par Richard Le Blanc (Paris: ]. Houzé, 1584)

- Hieronymi Cardani medici mediolanensis, De svbtilitate libri XXI (Paris: Michel Fezandat et Robert Granjon, 1550)

Celsus, Aurelius Cornelius, Avrelii Cor. Celsi de re medica libri octo (Lyon: Guillaume Roville, 1566)

Cicéron, Marcus Tullius Cicero, Cicero's Letters to Atticus, éd. D.R. Shackleton Bailey, vol. 5 (Cambridge: Cambridge University Press, 1966)

- Cicero: Letters to His Friends, éd. et trad. Glynn Williams, 3 vols, The Loeb Classical Library (Londres: William Heinemann; Cambridge, MA: Harvard University Press, première édition 1927-1929, plusieurs réimpressions)

Cinzio, Giovanni Battista Giraldi, «Discorso [...] intorno al comporre delle commedie, e delle tragedie», in Discorsi di M. Giovambattista Giraldi Cinthio nobile ferrarese... (Vinegia: Giolito, 1550)

Clairvaux, Bernard de, Sermons sur le Cantique, texte latin de J. Leclercq, H. Rochais et $\mathrm{Ch}$. H. Talbot; introduction, traduction et notes par Paul Verdeyen et Raffaele Fassetta; tome I, Sermons 1-15 (Paris: Éditions du cerf, 1996) 
Colonna, Francesco, Francesco Colonna, Le Songe de Poliphile, traduction de l'Hypnerotomachia Poliphili par Jean Martin (Paris: Kerver, 1546). Éd. Gilles Polizzi (s.l.: Imprimerie nationale Éditions, 1994)

- Hypnerotomachia Poliphili, Le Songe de Poliphile, fac-similé de la première édition française de 1546 avec les bois attribués à Jean Goujon d'après l'École de Mantegna, présenté par Albert-Marie Schmidt (Paris: Club des Libraires de France, 1963)

Crenne, Hélisenne de (probablement le pseudonyme de Marguerite Briet), Les Angoisses douloureuses qui procèdent d'amour, éd. Jean-Philippe Beaulieu (SaintÉtienne: Publications de l'Université de Saint-Étienne, 2005)

D’Aubigné, Agrippa, Pages inédites de Théodore-Agrippa d'Aubigné, éd. PierrePaul Plan (Genève: Société d'histoire et d'archéologie, 1945)

Dickens, Charles, Bleak House, éd. Norman Page (Londres: Penguin, 1971)

Du Bellay, Joachim, «The Regrets,» with «The Antiquities of Rome», Three Latin Elegies, and «The Defense and Enrichment of the French Language», édition bilingue, éd.et trad. par Richard Helgerson, PENN (Philadelphie: University of Pennsylvania Press, 2006)

- Divers Jeux rustiques, éd. V.L. Saulnier, Textes littéraires français (Paris: Minard; Genève: Droz, 1965)

Du Cange, Charles du Fresne, sieur du Cange, Glossarium mediae et infimae Latinitatis, 7 vols (Graz, [Autriche]: Akademische Druck- und Verlagsanstalt, 1954; publié pour la première fois en 1678)

Du Laurens, André, Discours de la conservation de la veuë: des maladies melancholiques: des catarrhes: \& de la vieillesse (Paris: T. Samson, 1598)

Du Pré, Jehan, Le Palais des nobles Dames, éd. Brenda Dunn-Lardeau, Textes de la Renaissance 115 (Paris: Champion, 2007)

- Le Palais des nobles Dames (Lyon [sans nom d'éditeur], 1534)

Estienne, Charles, L'Agriculture et maison rustique de M. Charles Estienne docteur en medecine [...], éd. Jean Liébault (Paris: Jacques Du-Puys, 1570)

Érasme, Didier (Erasmus Desiderius), Opera omnia Desiderii Erasmi Roterodami, section IV, vol. 2 (Amsterdam, Oxford: North-Holland, 1977) 
Fernel, Jean, La Physiologie, trad. du latin par Charles de Saint-Germain; texte revu par José Kany-Turpin, Corpus des Fuvres de philosophie en langue française ([Paris:] Fayard, 2001). Il s'agit d'une réédition de l'ouvrage édité à Paris, chez Jean Guignard Le Jeune, en 1655, sous le titre Les VII Livres de la Physiologie.

- Les Sept Livres de la therapeutique universelle (Paris: Jean Guignard père et fils, 1655)

- De abditis rerum causis [...]. Aeditio secunda (Paris: Jacob Dupuys, 1551)

Ferrand, Jacques, Traité de l'essence et guérison de l'amour, éd. Donald Beecher et Massimo Ciavolella, Textes de la Renaissance, 153 (Paris: Classiques Garnier, 2010)

- Traité de l'essence et guérison de l'amour ou De la mélancolie érotique, éd. Gérard Jacquin et Éric Foulon (Paris: Économica, 2001)

Ficin, Marsile, Marsile Ficin, Commentaire sur «Le Banquet» de Platon, «De l'Amour» - Commentarium in convivium Platonis, De amore, texte établi, traduit, présenté et annoté par Pierre Laurens. Les Classiques de l'humanisme, 14 (Paris: Les Belles Lettres, 2002)

- Marsile Ficin, «Les Trois Livres de la Vie», traduction de Guy Le Fèvre de la Boderie, revue par Thierry Gontier, Corpus des œuvres de philosophie en langue française (Paris: Fayard, 2000)

- Théologie platonicienne, éd. Raymond Marcel, 3 vols (Paris: Les Belles Lettres, 1964-1970)

- Commentaire sur le Banquet de Platon, éd. Raymond Marcel (Paris: Les Belles Lettres, 1956)

Flaubert, Gustave, Madame Bovary, éd. Claudine Gothot-Mersch, Classiques Garnier (Paris: Garnier, 1971)

- Bouvard et Pécuchet, éd. Éd. Maynial, Classiques Garnier (Paris: Garnier, 1965)

Folengo, Teofilo, Baldus, éd. Emilio Faccioli (Turin: Giulio Einaudi, 1989) 
Franc Archer de Baignollet, Le, éd. Lucie Polak, Le Franc Archier de Baignollet, suivi de deux autres monologues dramatiques, Textes Littéraires Français, 129 (Genève: Droz; Paris: Minard, 1966)

Galien, Claude, [CFuvres, Tome II] Exhortation à l'étude de la médecine; Art médical, éd. Véronique Boudon, Collection des Universités de France (Paris: Les Belles lettres, 2000)

Gautier, Théophile, Émaux et Camées, éd. Jean Pommier et Georges Matoré, Textes Littéraires Français (Lille: Giard; Genève: Droz, 1947)

Gesner ou Gessner, Conrad, Historiae Animalium (Zurich: Froschover, 1551-1585)

- De rerum fossilium, lapidum et gemmarum maximè, figuris et similitudinibus Liber (Zurich: Jacob Gesner, 1565)

Gohory, Jacques, Theophrasti Paracelsi philosophiae et medicinae utriusque universae compendium ex optimis quibuscumque ejus libris, cum scholiis in libros IIII ejusdem de vita longa [...] auctore Leone Suairo ].G. [Gohory] (Parisiis: in aedibus Rovillii [s.d.])

Greimas, Algirdas Julien et Teresa Mary Keane, Dictionnaire du moyen français: La Renaissance (Paris: Larousse, 1992)

Grévin, Jacques, La Trésorière; Les Esbahis: comédies, éd. E. Lapeyre, Société des Textes Français Modernes (Paris: Champion, 1980)

Guillet, Lucie, La Poéticothérapie: Guérir par la poésie. Efficacités du fluide poétique (Paris: Jouve, 1946)

Guillet-Laburthe, Suzanne, voir Macrin, Jean Salmon

Habert, François, Les Trois Livres de la Chrysopee c'est-à-dire l'art de faire l'or [...] traduicts de Jean Aurelle Augurel, poète latin, par F. Habert de Berry (Paris: ]. Longis, 1550)

Habert, Isaac, Les Trois Livres des meteores avecques autres œuures poëtiques (Paris: Jean Richer, 1585)

Hébreu, Juda Abravanel, dit Léon Hebreu, ou Leone Ebreo, Dialogues d'amour, traduction de Pontus de Tyard (1551) [en orthographe modernisée], «De Pétrarque à Descartes» (Paris: Vrin, 2006) 
Hesteau de Nuysement, Clovis, Les (Fuvres poétiques, Livre Ier, éd. Roland Guillot, Textes Littéraires Français 446 (Genève: Droz, 1994)

- Les Fuvres poétiques (Paris: Abel l'Angelier, 1578)

Horace, Quintus Horatius Flaccus, éd. et trad. James Michie, Penguin Classics (Harmondsworth: Penguin, 1967)

Huguet, Edmond, Dictionnaire de la langue française du seizième siècle, 7 vols (Paris: Édouard Champion, puis Didier, 1925-1967)

Jamot, Frederic, Traicté de la Goutte, contenant les Causes et origines d'icelle, le moyen de s'en pouvoir preserver Et la sçavoir guerir estant acquise. Escrit en grec du commandement de Michel Paleologue, Empereur de Constantinople par Demetrius Pepagomenus son premier medecin. Traduict en François [...] par M. Frederic Jamot, docteur en medecine (Paris: Ph. G. de Roville, 1567)

Jodelle, Estienne, L'Eugène, éd. de M.J. Freeman, Textes Littéraires, 65 (Exeter: University of Exeter, 1987)

Joubert, Laurent, Question des hvilles traictee problematiquement par M. Lavr. lovbert [...] Item, Censure de quelques opinions touchant la decoction pour les arquebuzades par le mesme auteur (Lyon: Benoist Rigaud, 1588)

- Traité $d v$ ris, contenant son essance, ses causes, et mervelheus effais (Paris: Nicolas Chesneau, 1579)

- Errevrs popvlaires av fait de la medecine et regime de sante (Avignon: Guillaume Bertrand, 1578)

- Traitté des Arcbvsades (Lyon: Jean de Tournes, 1574)

Kochanowski, Jan, La Vie qu'il faut choisir, traduit du polonais et du latin, et présenté par Alice-Catherine Carls (s.l.: Orphée / La Différence, 1992). Il s'agit d'une sélection de textes, avec la version originale en face de la traduction.

- Carmen macaronicum de eligendo vitae genere (Varsovie: Państwowy Instytut Wydawniczy, 1981)

La Boëtie, Estienne de, Discours de la servitude volontaire, éd. Simone GoyardFabre (Paris: Garnier-Flammarion, 1993) 
La Jessée, Jean de, Premieres CFuvres Françoises de Jean de La Jessee (Anvers: Christofle Plantin, 1583)

Landsberg, Herrad von (von Hohenbourg), Hortus deliciarum, éd. Rosalie Green et al., 2 vols, Studies of the Warburg Institute, 36 (Londres: Warburg Institute, 1979)

Lapidaires grecs, Les Lapidaires grecs: Lapidaire orphique, Kérygmes lapidaires d'Orphée; Socrate et Denys; Lapidaire nautique; Damigéron-Évax, éd. Robert Halleux et Jacques Schamp, Collection des Universités de France (Paris: Les Belles Lettres, 1985)

Larivey, Pierre de, Les Esprits, éd. M.J. Freeman (préface de Madeleine Lazard), Textes Littéraires Français (Genève: Droz, 1987)

La Rue, François, De Gemmis aliquot [...] (Paris: Chrestien Wechel, 1547)

Latham, R.E. (éd.), Revised Medieval Latin Word-List from British and Irish Sources (Londres: Oxford University Press, 1965; réimpr. 1973, 1980)

L'Estoile, Pierre de, Journal du règne de Henri III (1574-1589) (Paris: Lefèvre, 1943)

Lewis, Charlton T., et Charles Short, A Latin Dictionary Founded on Andrews' Edition of Freund's Latin Dictionary (Oxford: Oxford University Press, 2002; première édition 1879)

Longus, Les Amours pastorales de Daphnis et Chloé, éd. Sabine Wespieser, Les Belles Infidèles (Arles: Actes Sud, 1988)

- Pastorales (Daphnis et Chloé), texte établi et traduit par J.R. Vieillefond, Collection des Universités de France (Paris: Belles Lettres, 1987)

Machiavelli, Niccolò, The Comedies of Machiavelli: The Woman from Andros; The Mandrake; Clizia, éd. David Sices et James B. Atkinson, (Hanovre et Londres: University Press of New England, 1985)

- Clitia. Comedia facetissima di Clitia: composto per lo ingenioso huomo Nicolò Machiauelli fiorentino: nuouamente stampata ([Florence: sans nom d'éditeur] MDXXXVII [1537])

Macrin, Jean Salmon, Hymnes (1537), éd., traduction et commentaire par Suzanne Guillet-Laburthe, Travaux d'humanisme et Renaissance, 481 (Genève: Droz, 2010) 
Marbode de Rennes, éd. John M. Riddle, Marbode of Rennes' (1035-1123) «De lapidibus» Considered as a Medical Treatise With Text, Commentary and C.W. King's Translation Together With Text and Translation of Marbode's Minor Works on Stones (Wiesbaden: Franz Steiner, 1977)

Martin, Jean, L'Arcadie de Sannazar [...] mise d'Italien en François par lehan Martin, Secrétaire de Monseigneur [...] Cardinal de Lenoncourt (Paris: Michel de Vascosan et Gilles Corrozet, 1544)

Matthiole, Pierre André, Commentaires de M. Pierre André Matthiole medecin senois, [...] sur les six livres de Ped. Dioscoride [...] Reveuz et augmentés [...] par l'autheur mesme [...] mis en françois [...] par M. lean des Moulins Docteur en Medecine (Lyon: Guillaume Roville, 1572)

Montaigne, Michel de, Essais éd. par Jean Balsamo, Michel Magnien et Catherine Magnien-Simonin, Bibliothèque de la Pléiade, NRF (Paris: Gallimard, 2007)

- Journal de voyage, éd. Fausta Garavini, Folio (Paris: Gallimard, 1983)

Navarre, Marguerite de, L'Heptaméron, éd. de M. François, Classiques Garnier (Paris: Garnier, 1967)

Nicot, Jean, Thresor de la langue françoise, tant ancienne que moderne (Paris: David Douceur, 1621)

Ovide, Publius Ovidius Naso, Metamorphoseon Libri XV, éd. B.A. van Proosdij (Leiden: Brill, 1975)

Palissy, Bernard, Recepte veritable, éd. Keith Cameron, Textes Littéraires Français (Genève: Droz, 1988)

Paré, Ambroise, Euvres complètes, éd. J.-F. Malgaigne, 3 vols (Paris: J.-B. Baillière, 1840)

- CEvvres, 2 édition (Paris: Gabriel Buon, 1579)

- La Methode de traicter les playes faictes par hacquebvtes et avltres bastons à feu [...] (Paris: Gaulterot, 1545)

Pasquier, Estienne, Recherches de la France (Paris: Laurens Sonnius, 1621) 
Pétrarque, Francesco Petrarca, Lettres familières, trad. André Longpré, notice et notes de Ugo Dotti, 4 vols, Les Classiques de l'Humanisme (Paris: Les Belles Lettres, 2002-2004)

- Rerum familiarium, Lettres familières, trad. André Longpré (Paris: Les Belles Lettres, 2005)

Plaute, Titus Maccius, Plautus: The Comedies, Complete Roman Drama in Translation, éd. David R. Slavitt et Palmer Bovie, 4 vols (Baltimore et Londres: Johns Hopkins University Press, 1995)

- Casina, éd. W.Thomas MacCary et M.M. Willcock, Cambridge Greek and Latin Classics (Cambridge: Cambridge University Press, 1976)

Pline l'Ancien, Caius Plinius Secundus, Natural History X Books XXXVI-XXXVII, éd. et trad. D.E. Eichholz, Loeb Classical Library (Cambridge, MA: Harvard University Press; Londres: Heinemann, 1971)

Quinze, Les Quinze Joyes du mariage, éd. Joan Crow, Blackwell's French Texts (Oxford: Blackwell, 1969)

Ramonneurs, Les, comédie anonyme, éd. Austin Gill, Société des Textes Français Modernes (Paris: Didier, 1957)

Robortellus, Franciscus, Francisci Robortelli vtinensis in librum Aristotelis de arte poetica explicationes (Florence: in officina Laurentii Torrentini, 1548)

Ronsard, Pierre de, Euvres complètes, éd. Jean Céard, Daniel Ménager et Michel Simonin, Bibliothèque de la Pléiade, 2 vols (Paris: Gallimard, 1993-1994)

- Discours des misères de ce temps, éd. Malcolm Smith (Genève: Droz, 1979)

- Ronsard II: Odes, Hymns and Other Poems, éd. Grahame Castor et Terence Cave (Manchester: Manchester University Press, 1977)

- Sonnets pour Helene, éd. Malcolm Smith, TLF (Genève: Droz; Paris: Minard, 1970)

- Les Amours de Pierre de Ronsard, éd. Henri et Catherine Weber (Paris: Garnier, 1963)

- Fuvres complètes, éd. Paul Laumonier, Société des Textes Français Modernes, 20 vols (Paris: Hachette, puis Droz, puis Didier, 1914-1975) 
Sainctes, Claude de, évêque d'Évreux, Discours sur le saccagement des Eglises Catholiques par les Heretiques anciens, \& nouueaux Calvinistes, en l'an 1562, éd. L. Cimber et F. Danjou, Archives curieuses de l'histoire de France, $1^{\text {re }}$ série, t. IV (Paris: Beauvais, 1835)

- Discours sur le saccagement des Eglises Catholiques par les Heretiques anciens, \& nouueaux Calvinistes, en l'an 1562 (Verdun: N. Bacquenois, 1562)

Saint-Exupéry, Antoine de, Vol de nuit, Livre de Poche (Paris: Gallimard, 1931)

Sidney, Sir Philip, Sir Philip Sidney: An Apology for Poetry (or The Defence of Poesy), éd. R.W. Maslen (Manchester et New York: Manchester University Press, 2002; première edition 1965)

Stendhal, Henri Beyle, dit, Le Rouge et le Noir, éd. Henri Martineau, Classiques Garnier (Paris: Garnier, 1950)

Taillemont, Claude de, Discours des champz faëz (Lyon: Michel Du Boys, 1553)

Tissier, André (éd.), Recueil de farces (1450-1550), III (Genève: Droz, 1988); IV, Textes Littéraires Français 367 (Genève: Droz, 1989); V (Genève: Droz, 1989); VI (Genève: Droz, 1990); IX (Genève: Droz, 1995); et X, Textes Littéraires Français 471 (Genève: Droz, 1996)

Veyras, Jacques et Tannequin Guilhemet, Traicté de chirurgie, contenant la vraye methode de gverir playes d'arquebusade, selon Hippocras, Galen \& Paracelse, auec refutation des erreurs qui s'y commettent (Lyon: Berthelemy Vincent, 1581)

Virgile, Publius Vergilius Maro, Opera, éd. R.A.B. Mynors, Oxford Classical Texts (Oxford: Clarendon, 1969)

Wonnecke von Caub, Johann (Johannes de Cuba), Hortus sanitatis (MünchenAllach: Konrad Kölbl, 1966)

Yver, Jacques, Le Printemps d'Iver. Contenant cinq Histoires, discourues par cinq iournees, en vne noble compagnie, au chasteau du Printemps. Par laqves Yver seigneur de Plaisance, \& de la Bigottrie, gentilhomme Poicteuin. Tierce edition (Paris: Jean Ruelle, 1572)

Zweig, Stefan, Die Heilung durch den Geist. Mesmer - Mary Baker Eddy - Freud (Leipzig: Insel, 1931) paru en France sous le titre de La Guérison par l'esprit (Paris: LGF - Livre de Poche, 1932) 


\section{Sources secondaires}

Adams, J.N., The Latin Sexual Vocabulary (Londres: Duckworth, 1982; deuxième impression 1987; troisième impression 1990)

Adams, Robert P., The Better Part of Valor: More, Erasmus, Colet and Vives, on Humanism, War and Peace, 1496-1535 (Seattle: University of Washington Press, 1962)

Allen, Michael J.B., Nuptial Arithmetic: Marsilio Ficino's Commentary on the Fatal Number in Book VIII of Plato's 'Republic' (Berkeley - Los Angeles - Londres: University of California Press, 1994)

Amicis, Vincenzo de, L'Imitazione latina nelle commedia italiana del XVI secolo, Biblioteca critica della letteratura italiana diretta da Francesco Torraca (Florence: Sansoni, 1897)

Archambault, Paul, «The Analogy of the 'Body' in Renaissance Political Literature», Bibliothèque d'humanisme et Renaissance, 29 (1967), p. 21-53

Archibald, Elizabeth, «Tradition and Innovation in the Macaronic Poetry of Dunbar and Skelton», Modern Language Quarterly, 53 (1992), p. 126-149

Armstrong, Elizabeth, Ronsard and the Age of Gold (Cambridge: Cambridge University Press, 1968)

Ashworth, William B., Jr., «Emblematic Natural History of the Renaissance», in N. Jardine, J.A. Secord et E.C. Spary (éd.), Cultures of Natural History (Cambridge: Cambridge University Press, 1996), p. 17-37

Atkinson, James B., «An Essay on Machiavelli and Comedy», dans l'édition The Comedies of Machiavelli: The Woman from Andros; The Mandrake; Clizia, éd. et trad. par David Sices et James B. Atkinson, p. 1-34. Voir Machiavel dans les sources primaires.

Auer, P., «The Pragmatics of Code-Switching: A Sequential Approach», in One Speaker, Two Languages, éd. Milroy et Muysken, p. 115-135

Bakhtine, Mikhaïl, L'CFuvre de François Rabelais et la culture populaire au Moyen Âge et sous la Renaissance, trad. du russe par Andrée Robel (Paris: Gallimard, 1982, 1990) 
Balmas, Enea, Un Poeta del Rinascimento francese, Étienne Jodelle: La sua vita il suo tempo, Biblioteca dell'«Archivum romanicum», I, 66 (Florence: Olschki, 1962)

Frederic C. Bartlett, Remembering (Cambridge: Cambridge University Press, 1932)

Barraud, Georges, L'Humanisme et la médecine au XVle siècle (Paris: Vigot, 1942)

Baudry, Hervé, Contribution à l'étude du paracelsisme en France au XVI siècle (1560-1580). De la naissance du mouvement aux années de maturité: «Le Demosterion» de Roch le Baillif (1578), Études et Essais sur la Renaissance, 60 (Paris: Champion, 2005)

Baulant, Micheline, et Jean Meuvret, Prix des céréales extraits de la mercuriale de Paris (1520-1698), I, 1520-1620, École Pratique des Hautes Études, Sixième Section (s.l.: S.E.V.P.E.N., 1960)

Beaune, Colette, Naissance de la nation France (Paris: Gallimard, 1985\}

Beecher, Donald A., «Des Médicaments pour soigner la mélancolie: Jacques Ferrand et la pharmacologie de l'amour», Nouvelle Revue du Seizième Siècle, 4 (1986), 87-99

Bellenger, Yvonne, «Des Académies italiennes à celles de France au XVIe siècle», in Luisa Rotondi Secchi Tarugi (éd.), Rapporti e scambi tra umanesimo italiano ed umanesimo europeo: "L'Europa e' uno stato d'animo», Caleidoscopio, 10 (Milan: Nuovi Orizzonti, 2001), p. 11-22

- Le Jour dans la poésie française au temps de la Renaissance, Études littéraires françaises, 2 (Tübingen: Narr; Paris: Place, 1979)

- Du Bellay: Ses «Regrets» qu'il fit dans Rome (Paris: Nizet, 1975)

- La Journée et ses moments dans la poésie française du XVle siècle, thèse (Lille-Paris, 1975)

Bennet, Glin, The Wound and the Doctor: Healing, Technology and Power in Modern Medicine (Londres: Secker and Warburg, 1987)

Besser, Reinhold, «Über Remy Belleaus Steingedicht „Les Amours Et Nouveaux Eschanges Des Pierres Precieuses, Vertus Et Proprietez D'Icelles"», Zeitschrift für neufranzösische Sprache und Literatur, 8 (1886), p. 185-250 
Besserman, Lawrence L., The Legend of Job in the Middle Ages (Cambridge MA et Londres: Harvard University Press, 1979)

Białostocki, Jan, L'Art du XVe siècle des Parler à Dürer, traduit de l'anglais et de l'italien par Pierre-Emmanuel Dauzat, Encyclopédies d'aujourd'hui, La Pochothèque (Paris: Le Livre de Poche, 1993)

Bianchi, Massimo Luigi, Introduzione a Paracelso, I Filosofi (Rome et Bari: Laterza, 1995)

Bichard-Thomine, Marie-Claire, Noël du Fail Conteur, Études et essais sur la Renaissance, 28 (Paris: Champion, 2001)

Bizer, Marc, La Poésie au miroir: Imitation et conscience de soi dans la poésie latine de la Pléiade (Paris: Champion, 1995)

Blanchard, Joël, La Pastorale en France aux XIVe et XV siècles: Recherches sur les structures de l'imaginaire médiéval, Bibliothèque du XV siècle, 45 (Paris: Champion, 1983)

Bloch, Marc, Les Rois thaumaturges (Paris: Gallimard, 1983; publié pour la première fois en 1924)

Bolzoni, Lina, La Stanza della memoria: modelli letterari e iconografici dell'età della stampa (Turin: Einaudi, 1995), traduit par Marie France Berger, La Chambre de la mémoire: Modèles littéraires et iconographiques à l'âge de l'imprimerie (Genève: Droz, 2005)

- «L'Art de la mémoire et le travail de l'oubli», dans Mémoire et oubli au temps de la Renaissance. Actes du colloque de Paris 8-9 décembre 2000 et 9-10 mars 2001, éd. M.T. Jones-Davies (Paris: Champion, 2002), p. 145-157

Boucher, Jacqueline, Société et mentalités autour de Henri III, thèse présentée devant l'Université de Lyon II - le 22 octobre 1977, 4 vols (Lille: Atelier Reproduction des thèses, Univ. de Lille III; Paris: Diffusion Champion, 1981), III, p. 891. Ouvrage publié depuis en un volume (Paris: Champion, 2007)

Boughner, Daniel C., The Braggart in Renaissance Comedy: A Study in Comparative Drama from Aristophanes to Shakespeare (Minneapolis: University of Minnesota Press [1954])

Bowen, Barbara C., Barbara C. Bowen, «Rabelais's Unreadable Books», Renaissance Quarterly, 48 (1995), p. 742-758 
- Les Caractéristiques essentielles de la farce française et leur survivance dans les années 1550-1620, Illinois Studies in Language and Literature, 53 (Urbana: University of Illinois Press, 1964)

Brabant, H., Médecins, malades et maladies de la Renaissance, La Lettre et l'esprit (Bruxelles: La Renaissance du Livre, 1966)

Braun, Lucien, Conrad Gessner, Les Grands Suisses (Genève: Slatkine, 1990)

Braybrook, Jean, «Réflexions sur Les Amours de David et de Bersabée, de Remy Belleau», in Renaissance Reflections: Essays in Memory of C.A. Mayer, éd. Pauline M. Smith et Trevor Peach, La Renaissance française, 10 (Paris: Champion, 2002), p. $161-172$

- «Remy Belleau's La Reconnue and Niccolò Machiavelli's Clizia», Renaissance Studies, 15 (2001), p. 1-16

- «Remy Belleau's Macaronic Poem, De Bello Huguenotico, and the French Wars of Religion», in Poets and Teachers: Latin Didactic Poetry and the Didactic Authority of the Latin Poet from the Renaissance to the Present, éd. Yasmin Haskell et Philip Hardie, Kleos, Estemporaneo di studi e testi sulla fortuna dell'antico, a cura di Francesco de Martino, 4, 1999 (Bari: Levante, 1999), p. 183-198

- «Space and Time in Remy Belleau's Bergerie», Bibliothèque d'humanisme et Renaissance, 57 (1995), 369-380

- «Science and Myth in the Poetry of Remy Belleau», Renaissance Studies, 5 (1991), 277-287

- «The Curative Properties of Remy Belleau's Pierres Precieuses», in Explorations in Renaissance Culture, 16 (1990)

- «Remy Belleau and the Pierres precieuses» in Renaissance Studies, 3 (1989), 193-201

- «Remy Belleau et les pierres précieuses de l'Apocalypse» in Bibliothèque d'Humanisme et Renaissance, 51 (1989), p. 405-406

- «Les Sonnets pétrarquistes de Remy Belleau», in Le Sonnet à la Renaissance, éd. Yvonne Bellenger (Paris, Aux Amateurs de Livres, 1988), p. $167-180$ 
- «Remy Belleau and the Figure of the Artist», French Studies, 37 (1983), p. 1-16

- «The Epic Fragment in Mid Sixteenth-Century French Poetry», these de doctorat (Oxford, 1981)

Brockliss, Laurence et Colin Jones, The Medical World of Early Modern France (Oxford: Clarendon Press, 1997)

Brooks, Jeanice, Courtly Song in Late Sixteenth-Century France (Chicago: University of Chicago Press, 2000)

Brown, Cinthia ]., «Mémoire et histoire: La Déformation de la réalité chez les Rhétoriqueurs à la fin du Moyen Âge», in Jeux de mémoire: Aspects de la mnémotechnie médiévale, recueil d'études publié sous la direction de Bruno Roy et Paul Zumthor (Montréal: Presses de l'Université de Montréal; Paris: Vrin, 1985), p. 43-53

Brunn, Alain, Le Laboratoire moraliste, Les Littéraires (Paris: PUF, 2009)

Cameron, Euan, The European Reformation (Oxford: Clarendon Press, 1991)

Campbell, Thomas P., éd., Tapestry in the Renaissance: Art and Magnificence (New York: The Metropolitan Museum of Art; New Haven et Londres: Yale University Press, 2002)

Campo, Roberto E., «Du miroir à la mémoire: sur les jeux ecphrastiques de $L a$ Bergerie de Remy Belleau», Nouvelle Revue du Seizième Siècle, 20 (2002), 5-23

Cannella, Anne-Françoise, Gemmes, verre coloré, fausses pierres précieuses au Moyen Âge: le quatrième livre du «Trésorier de Philosophie naturelle des pierres précieuses» de Jean d'Outremeuse, Bibliothèque de la Faculté de Philosophie et Lettres de l'Université de Liège, 288 (Genève: Droz, 2006)

Capitani, Patrizia de, Du Spectaculaire à l'intime: Un Siècle de commedia erudita en Italie et en France. Début XVIe siècle - milieu du XVIle siècle, Bibliothèque Littéraire de la Renaissance, 56 (Paris: Champion, 2005)

Carel, Havi, Illness: The Cry of the Flesh, The Art of Living (Durham: Acumen, 2008)

Carlino, Andrea, et Michel Jeanneret, éd., Vulgariser la médecine: Du Style médical en France et en Italie (XVI et XVII siècles), Cahiers d'humanisme et Renaissance, 89 (Genève: Droz, 2009) 
Carnel, Marc, Le Sang embaumé des roses: Sang et passion dans la poésie amoureuse de Pierre de Ronsard, Travaux d'humanisme et Renaissance, 395, Études Ronsardiennes, 10 (Genève: Droz, 2004)

Carruthers, Mary, The Book of Memory: A Study of Memory in Medieval Culture (Cambridge: Cambridge University Press, 1994; publié pour la première fois en 1990), traduit par Diane Meur, Le Livre de la mémoire: Une Étude de la mémoire dans la culture médiévale (Paris: Macula, 2002)

- The Craft of Thought: Meditation, Rhetoric and the Making of Images 4001200, Cambridge Studies in Medieval Literature, 34 (Cambridge: Cambridge University Press, 1998), traduit par Fabienne Durand Bogaert, Machina memorialis: Méditation, rhétorique et fabrication des images au Moyen Âge, Bibliothèque des Histoires (Paris: Gallimard, 2002)

Castiglioni, Arturo, A History of Medicine, traduit de l'italien par E.B. Krumbhaar (New York: Alfred A. Knopf, 1947)

Cave, Terence C., The Cornucopian Text: Problems of Writing in the French Renaissance (Oxford: Clarendon, 1979), p. 256-268. Ce livre a été traduit en français par Ginette Morel sous le titre: Cornucopia. Figures de l'abondance au XVIe siècle: Érasme, Rabelais, Ronsard, Montaigne (Paris: Macula, 1997)

- Recognitions: A Study in Poetics (Oxford: Clarendon, 1988)

- Devotional Poetry in France c. 1570-1613 (Cambridge: Cambridge University Press, 1969)

Cazilhac, Évelyne, «Un Bain biblique ou Bethsabée femme symbole: de pierre, de chair et d'eau (du XVe à la fin du XVIe siècle)», in Sources et fontaines du Moyen Age à l'Age baroque, Actes du Colloque tenu à l'Université Paul-Valéry (Montpellier III) les 28, 29 et 30 novembre 1996. Équipe d'Accueil Moyen Age - Renaissance - Baroque. Colloques, congrès et conférences sur la Renaissance, 12 (Paris: Champion, 1998), p. 221-231

Céard, Jean, (éd.), La Curiosité à la Renaissance: actes [de la seconde Journée d'études de la] Société française des seiziémistes [Paris, 16 mai 1981] (Paris: Société d'édition d'enseignement supérieur, 1986)

- La Nature et les prodiges: L'insolite au XVI siècle, en France, Travaux d'humanisme et Renaissance, 158 (Genève: Droz, 1977; réédité en 1995 avec une nouvelle préface de Céard) 
Cerquiglini-Toulet, Jacqueline, La Couleur de la mélancolie: La Fréquentation des livres au XIVe siècle 1300-1415 (Paris: Hatier, 1993)

Chamard, Henri, Histoire de la Pléiade, 4 vols (Paris: Didier, 1939-1940)

Champion, Pierre, «Henri III et les écrivains de son temps», Bibliothèque d'humanisme et Renaissance, 1 (1941), 43-172

Chastel, André, Marsile Ficin et l'art, Travaux d'humanisme et Renaissance, 14 (Genève: Droz, 1954 et 1975)

Chayes, Evelien, L'Éloquence des Pierres précieuses: De Marbode de Rennes à Alard d'Amsterdam et Remy Belleau. Sur quelques lapidaires du XVIe siècle (Paris: Champion, 2010)

Chevrolet, Teresa, L'Idée de fable: Théories de la fiction poétique à la Renaissance, Travaux d'humanisme et Renaissance, 423 (Genève: Droz, 2007)

Cloutier, Marilynn Roach, «Remy Belleau's Petites Inventions of 1556: A Generic and Stylistic Study» (PhD, University of Wisconsin-Madison, mai 1976)

Connat, M. et J. Mégret, «Mort et testament de Remy Belleau», Bibliothèque d'humanisme et Renaissance, 6 (1945), 328-356

Constant, Jean-Marie, Les Guise ([Paris:] Hachette, 1984)

Corbin, Alain, Jean-Jacques Courtine, Georges Vigarello (éd.), Histoire du corps, 3 volumes, I, De la Renaissance aux Lumières, vol. dirigé par Georges Vigarello, L'Univers historique (Paris: Seuil, 2005)

Couchman, Jane, «Les Eclogues sacrées prises du Cantique des Cantiques de Salomon et la poésie biblique de Remy Belleau», Renaissance and ReformationRenaissance et Réforme, 11 (1987), 29-39 (p. 36-37)

Crépet, Eugène (éd.), Les Poëtes français, recueil des chefs-d'œuvre de la poésie française depuis les origines jusqu'à nos jours [...], 4 vols (Paris: Gide, 1861-1863)

Csürös, Klára, «Sainte sagesse et diabolique démesure d'un roi: Les Amours de David et de Bersabée (1572), épyllion de Rémi Belleau», in Héroïsme et démesure dans la littérature de la Renaissance: Les Avatars de l'épopée, éd. Denise Alexandre (Saint-Étienne: Publications de l'Université de Saint-Étienne, 1998), p. 165-179 
Curtius, Ernst Robert, European Literature and the Latin Middle Ages, traduit de l'allemand par Willard R. Trask, Bollingen Series 36 (Princeton: Princeton University Press, 1967; première édition 1953). Ce texte parut d'abord sous le titre Europäische Literatur und lateinisches Mittelalter (Berne: A. Francke, 1948)

Dale, Hilda, «Remy Belleau et la science lapidaire», in Lumières de la Pléiade Neuvième stage international d'études humanistes, Tours, 1965, De Pétrarque à Descartes, 11 (Paris: Vrin, 1966), p. 231-242

Danese, Roberto M., «Casina, Clizia e la lora fortuna nel Cinquecento», in Lecturae plautinae sarsinates VI, Casina, éd. Renato Raffaelli et Alba Tontini (Urbino: QuattroVenti, 2003), p. 91-123

Dauvois, Nathalie, De la «Satura» à la Bergerie: Le Prosimètre pastoral en France à la Renaissance et ses modèles, Études et Essais sur la Renaissance, 22 (Paris: Champion, 1998)

- Mnémosyne: Ronsard, une poétique de la mémoire, Études et Essais sur la Renaissance, 1 (Paris: Champion, 1992)

Davis, Natalie Zemon, The Gift in Sixteenth-Century France (Oxford: Oxford University Press, 2000)

De Amicis, Vincenzo, voir Amicis, Vincenzo de

Delacourcelle, Doris, Le Sentiment de l'art dans la «Bergerie» de Remy Belleau (Oxford: Basil Blackwell, 1945)

Delcourt, Marie, La Tradition des comiques anciens en France avant Molière, Bibliothèque de la Faculté de Philosophie et de Lettres de l'Université de Liége [sic], 59 (Liège: Faculté de Philosophie et Lettres; Paris, Droz, 1934)

Deloffre, Frédéric, «Heurs et malheurs du temps: Une déploration macaronique de Rémy Belleau», in Il tema della fortuna nella letteratura francese e italiana del Rinascimento, Mélanges offerts à Enzo Guidici (Florence: Olschki, 1990)

Delumeau, Jean (éd.), La Mort des pays de Cocagne: Comportements collectifs de la Renaissance à l'âge classique, Publications de la Sorbonne, Série «Études», tome 12 (Paris: Sorbonne, 1976)

Demerson, Guy, «Belleau au travail: l'exemple de Sapphô» in Les Fruits de la Saison: Mélanges de littérature des XVI et XVII siècles offerts au Professeur 
André Gendre, éd. Philippe Terrier, Loris Petris et Marie-Jeanne Liengme Bessire (Neuchâtel: Université de Neuchâtel; Genève: Droz, 2000), p. 275-287

- «'Poétique de la réflexivité': Belleau face à Bèze et à Ronsard», in «Sans autre guide», Mélanges offerts à Marcel Tetel (Paris: Klincksieck, 1999), p. 83-95

- «Rabelais côté jardin», in Conteurs et romanciers de la Renaissance, Mélanges offerts à Gabriel-André Pérouse, Études recueillies et présentées par James Dauphiné et Béatrice Périgot, Colloques, congrès et conférences sur la Renaissance, 7 (Paris: Champion, 1997), p. 151-171

- «Poétique de la métamorphose chez Remy Belleau», in Poétiques de la métamorphose, Institut d'Études de la Renaissance et de l'Âge classique (SaintÉtienne: Publications de l'Université de Saint-Étienne, 1981), p. 125-142

- La Mythologie classique dans l'œuvre lyrique de la «Pléiade», Travaux d'humanisme et Renaissance, 119 (Genève: Droz, 1972)

Deramaix, Marc, Perrine Galand-Hallyn, Ginette Vagenheim et Jean Vignes, éd., Les Académies dans l'Europe humaniste: Idéaux et pratiques, Actes du colloque international de Paris, 10-13 juin 2003, éd. Marc Deramaix, Perrine GalandHallyn, Ginette Vagenheim et Jean Vignes, Travaux d'humanisme et Renaissance, 441 (Genève: Droz, 2008)

Desbordes, Françoise, La Rhétorique antique, Hachette supérieur (Paris: Hachette, 1996)

Desportes, Philippe, Les premieres œevvres de Philippes Des Portes. Au Roy de France et de Pologne (Paris: Robert Estienne, 1575)

Dewaele, Jean-Marc, Emotions in Multiple Languages (Basingstoke: Palgrave Macmillan, 2010)

Dez, Pierre, Histoire des Protestants et des Églises réformées du Poitou, I (La Rochelle: Imprimerie de l'Ouest, 1936)

Dinter, Martin T., Anatomizing 'Civil War': Studies in Lucan's Epic Technique (Ann Arbor, Michigan: University of Michigan Press, 2013)

Donaldson-Evans, Lance K., Love's Fatal Glance: A Study of Eye Imagery in the Poets of the École lyonnaise, Romance Monographs, 39 (Jackson, Mississippi: University of Mississippi Press, 1980) 
Dormandy, Thomas, The Worst of Evils: The Fight Against Pain (New Haven et Londres: Yale University Press, 2006)

Dubois, Claude-Gilbert, éd., L'Invention au XVIe siècle: textes recueillis et présentés par Claude-Gilbert Dubois (Bordeaux: Presses Universitaires de Bordeaux, 1987)

- Mythe et langage au seizième siècle, Collection Ducros, 8 (Bordeaux: Ducros, 1970)

Duffin, Christopher John, «Alectorius: The Cock's Stone», Folklore, 118 (2007), 325-341

- «Stones for the Stone: Minerals and Fossils in the Treatment of Renal Calculi», Pharmaceutical Historian, 36 (2006), 55-60

- «The Western Lapidary Tradition in Early Geological Literature: Medicinal and Magical Minerals», Geology Today, 21 (2005), 58-63

Dumaître, Paule, Ambroise Paré: chirurgien de quatre rois de France (Paris: Perrin, 1986)

Duport, Danièle, Le Jardin et la nature: Ordre et variété dans la littérature de la Renaissance (Genève: Droz, 2002)

- «Les Jardins qui sentent le sauvage»: Ronsard et la poétique du paysage, Cahiers d'humanisme et Renaissance, 57 (Genève: Droz, 2000)

Durling, Richard J., A Catalogue of Sixteenth Century Printed Books in the National Library of Medicine (US: Department of Health, Education, and Welfare, 1967)

Eckhardt, Alexandre, Remy Belleau, sa vie - sa «Bergerie»: Étude historique et critique (Budapest: Németh, 1917; Genève: Slatkine Reprints, 1969)

Engammare, Max, «David côté jardin: Bethsabée, modèle et anti-modèle à la Renaissance», in Cité des hommes, cité de Dieu: Travaux sur la littérature de la Renaissance en l'honneur de Daniel Ménager, éd. François Rouget, Marie-Christine Gomez-Géraud, Michel Magnien, Travaux d'humanisme et Renaissance, 375 (Genève: Droz, 2003), p. 533-542

- «La Morale ou la beauté? Illustrations des amours entre David et Bethsabée (II Sam. 11-12) dans les bibles des XVe-XVIIe siècles», in La Bible imprimée dans l'Europe moderne, sous la direction de Bertram Eugene Schwarzbach (Paris: Bibliothèque nationale de France, 1999), p. 447-476 
- «Qu'il me baise des baisiers de sa bouche»: Le Cantique des Cantiques à la Renaissance: Étude et bibliographie, Travaux d'humanisme et Renaissance, 277 (Genève: Droz, 1993)

Faisant, Claude, «Gemmologie et imaginaire: les Pierres precieuses de Remy Belleau» in L'Invention au XVI siècle: textes recueillis et présentés par ClaudeGilbert Dubois (Bordeaux: Presses Universitaires de Bordeaux, 1987), p. 83-106

Feldmann, Carsten, Rerum natura: Lukrez, Belleau, Ponge (Frankfurt am Main: Peter Lang, 1997)

Fernando, Diana, The Dictionary of Alchemy: History, People, Definitions (Londres: Vega, 2002)

Fest, Otto, Der Miles Gloriosus in der französischen Komödie vom Beginn der Renaissance bis zu Molière, Münchener Beiträge zur romanischen und englischen Philologie, 13 (Erlangen, Leipzig: Georg Böhme, 1897)

Festugière, A.-J., La Philosophie de l'amour de Marsile Ficin et son influence sur la littérature française au XV/e siècle (Paris: Vrin, 1941)

Field, J.V. et Frank A.J.L. James, éd, Renaissance and Revolution: Humanists, Scholars, Craftsmen and Natural Philosophers in Early Modern Europe (Cambridge: Cambridge University Press, 1993)

Flèges, Amaury, «']e ravie le mort', tombeaux littéraires en France à la Renaissance», dans Dominique Moncond'Huy, Le Tombeau poétique en France (Poitiers: La Licorne, UFR Langues Littératures Poitiers, 1994), p. 71-142

Fontaine, M.M., «Poète malade», in Libertés et savoirs du corps à la Renaissance (Caen: Paradigme, 1993), p. 243-261

- «Jean Martin, traducteur», in Prose et prosateurs de la Renaissance, Mélanges offerts à Robert Aulotte (Paris: SEDES, 1988), p. 109-122

Ford, Philip, De Troie à Ithaque: réception des épopées homériques à la Renaissance, Travaux d'humanisme et Renaissance, 436 (Genève: Droz, 2007)

Ford, Philip et Neil Kenny (éd.), La Librairie de Montaigne. Proceedings of the Tenth Cambridge French Renaissance Colloquium 2-4 September 2008 (Cambridge: Cambridge French Colloquia, 2012) 
Forestier, Georges, Esthétique de l'identité dans le théâtre français (1550-1680): Le Déguisement et ses avatars, Histoire des idées et critique littéraire, 259 (Genève: Droz, 1988)

- «Situation du personnage de la jeune fille dans la comédie française du XVIe siècle», Bibliothèque d'humanisme et Renaissance, 46 (1984), 7-19

Forster, Leonard, The Icy Fire: Five Studies in European Petrarchism (Cambridge: Cambridge University Press, 1969)

Foster, Kenelm, Petrarch Poet and Humanist, Writers of Italy, 9 (Edinburgh: Edinburgh University Press, 1987; première édition 1984)

Fowler, Alastair, Renaissance Realism: Narrative Images in Literature and Art (Oxford: Oxford University Press, 2003)

Fragonard, M.-M, La Pensée religieuse d'Agrippa d'Aubigné et son expression (Paris: Didier, 1986)

Fumaroli, Marc, L'Âge de l'éloquence: Rhétorique et «res literaria» de la Renaissance au seuil de l'époque classique (Genève: Droz, 1980; deuxième édition Paris: Albin Michel, 1994)

Galand-Hallyn, Perrine, Le Reflet des fleurs: Description et métalangage poétique d'Homère à la Renaissance, Travaux d'humanisme et Renaissance, 283 (Genève: Droz, 1994)

Garapon, Robert, La Fantaisie verbale et le comique dans le théâtre français du Moyen Âge à la fin du XVIIe siècle (Paris: A. Colin, 1957)

Gardner-Chloros, Penelope, Language Selection and Switching in Strasbourg (Oxford: Clarendon, 1991)

Genthe, F.W., Geschichte der Macaronischen Poesie, und Sammlung ihrer vorzüglichsten Denkmale (Halle et Leipzig: Reinicke et Compagnie, 1829)

Giry-Deloison, Charles et Roger Mettam, Patronages et clientélismes 15501750 (France, Angleterre, Espagne, Italie), Histoire et Littérature régionales, 10 (Villeneuve d'Ascq: Université Charles de Gaulle (Lille III); Londres: Institut français du Royaume-Uni, s.d.)

Glauser, Alfred, Le Poème-Symbole; De Scève à Valéry (Paris: Nizet, 1967) 
Godwin Joscelyn, The Pagan Dream of the Renaissance (Grand Rapids, MI: Phanes Press, 2002)

Grafton, Anthony, Cardano's Cosmos: The Worlds and Works of a Renaissance Astrologer (Cambridge, MA et Londres: Harvard University Press, 1999)

Graves, Robert, The Crowning Privilege: The Clark Lectures, 1954-1955 (Londres: Cassell, 1955; New York: Doubleday, 1956)

Grazia, Sebastian de Grazia, Machiavell in Hell (Londres et Basingstoke: Papermac, 1996; première édition 1989)

Griffiths, Richard, The Dramatic Technique of Antoine de Montchrestien: Rhetoric and Style in French Renaissance Tragedy (Oxford: Clarendon Press, 1970)

Guichemerre, Roger, La Comédie avant Molière: 1640-1660 (Paris: PUF, 1972)

Hall, James, Dictionary of Subjects and Symbols in Art (Londres: John Murray, 1979; première édition 1974)

Hampton, Timothy, Literature and Nation in the Sixteenth Century: Inventing Renaissance France (Ithaca et Londres: Cornell University Press, 2001)

Hartog, François, «Mémoire, histoire et lieux de mémoire», Memoria e memorie, Convegno internazionale di studi, Roma, 18-19 maggio 1995, Accademia Nazionale dei Lincei, éd. Lina Bolzoni, Vittorio Erlindo, Marcello Morelli, Centro Internazionale di Cultura «Giovanni Pico della Mirandola», Studi Pichiani, 6 ([Florence:] Olschki, 1998), p. 87-106

Harvey, Howard Graham, The Theatre of the Basoche: The Contribution of the Law Societies to French Mediaeval Comedy, Harvard Studies in Romance Languages, 17 (Cambridge, MA: Harvard University Press, 1941)

Hausen, Adelheid, Hiob in der französischen Literatur: Zur Rezeption eines alttestamentlichen Buches, Europäische Hochschulschriften, Reihe XIII, Band 17 (Berne: Herbert Lang, et Frankfurt-am-Main: Peter Lang, 1972)

Heller, Monica (éd.), Codeswitching: Anthropological and Sociolinguistic Perspectives (Berlin: Mouton de Gruyter, 1988)

Herrick, M.T., Italian Comedy in the Renaissance (Urbana et Londres: University of Illinois Press, 1966; première impression 1960) 
Higman, F.M., Piety and the People: Religious Printing in French 1511-1551 (Aldershot: Scolar press, 1996)

Hodges, Elisabeth, «Representing Place in Corrozet's Antiquitez de Paris», French Studies, 62 (2008), 135-149

Hoffmann, Hans, Hochrenaissance, Manierismus, Frühbarock: Die italienische Kunst des 16. Jahrhunderts (Zurich et Leipzig: Leemann, 1938)

Holmes, Urban Tigner, Jr., «The Background and Sources of Belleau's Pierres Précieuses», P.M.L.A. 61 (1946), p. 624-635

Hunkeler, Thomas, «Le Vif du sens»: Corps et poésie selon Maurice Scève, Cahiers d'humanisme et Renaissance, 66 (Genève, Droz, 2003)

Hunter, R.L., The New Comedy of Greece and Rome (Cambridge: Cambridge University Press, 1985)

Hutson, Lorna, The Userer's Daughter: Male Friendship and Fictions of Women in Sixteenth-Century England (Londres: Routledge, 1994)

James Hutton, «Cupid and the Bee», in Rita Guerlac (éd.), Essays on Renaissance Poetry, James Hutton (Ithaca et Londres: Cornell University Press, 1980), p. 106131

- «The Classics in Sixteenth-Century France», ibid., p. 207-224; première version dans The Classical Weekly, 43 (1950), p. 131-138

Jacquemier, Myriam, «L'Influence de l'Académie florentine dans le projet de l'Académie de musique et poèsie [sic] de Baïf», in Luisa Rotondi Secchi Tarugi (éd.), Rapporti e scambi tra umanesimo italiano ed umanesimo europeo: «L'Europa $e^{\prime}$ uno stato d'animo», Caleidoscopio, 10 (Milan: Nuovi Orizzonti, 2001), p. 65-84

Jardine, Lisa, Worldly Goods: A New History of the Renaissance (Londres: Macmillan, 1996)

Jeanneret, Michel, Perpetuum mobile: Métamorphoses des corps et des œuvres de Vinci à Montaigne, Argô (Paris: Macula, 1997)

- Des Mets et des Mots: Banquets et propos de table à la Renaissance (Paris: Corti, 1987) 
- Poésie et tradition biblique au XVI siècle: Recherches stylistiques sur les paraphrases des psaumes de Marot à Malherbe (Paris: Corti, 1969)

- «Les Euvres d'art dans La Bergerie de Belleau», Revue d'histoire littéraire de la France, 70 (1970), p. 1-13

Jeffery, Brian, French Renaissance Comedy 1552-1630 (Oxford: Clarendon Press, 1969)

Jouanna, Arlette, «Des Réseaux d'amitié aux clientèles centralisées: Les Provinces et la cour (France, XVIe et XVII siècle)», in Patronages et clientélismes 1550-1750 (France, Angleterre, Espagne, Italie), éd. Charles Giry-Deloison et Roger Mettam, Histoire et Littérature régionales, 10 (Villeneuve d'Ascq: Université Charles de Gaulle (Lille III); Londres: Institut français du Royaume-Uni, s.d.), p. 21-38

- Arlette Jouanna, La France du XVle siècle, 1483-1598, Collection Premier Cycle (Paris: PUF, 1997, deuxième édition corrigée; première édition 1996)

Joukovsky, Françoise, avec des dessins de Pierre Joukovsky, Le bel objet: Les paradis artificiels de la Pléiade, Confluences - Champion, 1 (Paris: Champion, 1991)

- «La Composition de la Bergerie de R. Belleau», in La Pastorale française: De Rémi Belleau à Victor Hugo, éd. Alain Niderst, Centre d'Étude et de Recherche d'Histoire des Idées et de la Sensibilité de l'Univ. de Rouen (Paris - Seattle - Tübingen, Biblio 17-63, 1991), p. 9-22

- La Gloire dans la poésie française et néolatine duXVle siècle (Des Rhétoriqueurs à Agrippa d'Aubigné), Travaux d'humanisme et Renaissance, 102 (Genève: Droz, 1969)

- «La Guerre des dieux et des géants chez les poètes français du XVle siècle (1500-1585)», Bibliothèque d'humanisme et Renaissance, 29 (1967), p. 5592

Kahn, Didier, Alchimie et Paracelsisme en France à la fin de la Renaissance (15671625), Cahiers d'humanisme et Renaissance, 80 (Genève: Droz, 2007)

Kahn, Victoria, «The Figure of the Reader in Petrarch's Secretum», in Petrarch, Modern Critical Views, éd. Harold Bloom (New York, Philadelphie: Chelsea House, 1989) 
Kennedy, George A., Classical Rhetoric and Its Christian and Secular Tradition from Ancient to Modern Times (Londres: Croom Helm, 1980, et Chapel Hill: University of North Carolina Press, 1980, réimpr. 1999)

Kenny, Neil, The Uses of Curiosity in Early Modern France and Germany (Oxford: Oxford University Press, 2004)

- Curiosity in Early Modern Europe: Word Histories, Wolfenbütteler Forschungen, 81 (Wiesbaden: Harrassowitz, 1998)

Kiple, Kenneth F., The Cambridge World History of Human Disease (Cambridge: Cambridge University Press, 1993, réimpr. 1994)

Kirkland, Ann Sorey, «Metamorphosis and Reflexivity in the Poetry of Belleau», Dissertation submitted in partial fulfilment of requirements for DPhil. in Department of Romance Studies in Graduate School of Duke University, supervisor Marcel Tetel, 1989

Knecht, R.J., The Rise and Fall of Renaissance France 1483-1610 (Londres: Fontana, 1996)

Kolbet, Paul R., Augustine and the Cure of Souls: Revising a Classical Ideal, Christianity and Judaism in Antiquity (Notre Dame, IN: University of Notre Dame Press, 2010)

Kremers, Edward, et George Urdang, History of Pharmacy, quatrième édition, révisée par Glenn Sonnedecker (Philadelphie, Toronto: J.B. Lippincott, 1976)

Kretzulesco-Quaranta, Emanuela, Les Jardins du songe: Poliphile et la mystique de la Renaissance (Rome: Editrice Magma; Paris: Les Belles Lettres, 1976)

Kuin, Roger, et Anne Lake Prescott, «The Wrath of Priapus: Rémy Belleau's 'Jean qui ne peult' and its Traditions», Comparative Literature Studies, 37 (2000), 1-17

La Charité, Raymond C., «Rabelais: The Book as Therapy», in Medicine and Literature, éd. Enid Rhodes Peschel (New York: Neale Watson Academic Publications, 1980), p. 11-17

Lambert, Ellen Zetzel, Placing Sorrow: A Study of the Pastoral Elegy Convention from Theocritus to Milton, University of North Carolina Studies in Comparative Literature, 60 (Chapel Hill: University of North Carolina Press, 1976) 
Langer, Ullrich, Perfect Friendship: Studies in Literature and Moral Philosophy from Boccaccio to Corneille, Histoire des Idées et Critique littéraire, 331 (Genève: Droz, 1994)

Lavillatte, Bruno, «Lire le corps comme on lit une carte: l'anatomie 'géographique' de la Physiologie de Jean Fernel», Revue des Amis de Ronsard, 24 (2011), p. 43-57

Lazard, Madeleine, Le Théâtre en France au XVle siècle (Paris: PUF, 1980)

- «Comédiennes et rôles féminins dans la comédie française du XVIe siècle», in Mélanges à la mémoire de Franco Simone: France et Italie dans la culture européenne, I: Moyen Âge et Renaissance (Genève: Slatkine, 1980)

- La comédie humaniste au XVle siècle et ses personnages (Paris: PUF, 1978)

Lebègue, Raymond, «Une Source de la Bergerie de Remy Belleau», Revue du Seizième Siècle, 4 (1916), p. 166-194

Lenient, C., La Satire en France ou la littérature militante au XVIe siècle, 2 vols (3e éd. Paris: Hachette, 1886, $1^{\text {re éd. } 1877)}$

Le Roux, Nicolas, La Faveur du roi: mignons et courtisans au temps des derniers Valois (vers 1547 - vers 1589), Époques (Seyssel: Champ Vallon, 2000)

Lestringant, Frank, «Les Amours pastorales de Daphnis et Chloé; fortunes d'une traduction de J. Amyot», in Fortunes de Jacques Amyot: Actes du colloque international (Melun 18-20 avril 1985), présentés par Michel Balard (Paris: Nizet, 1986), p. 237-257

Lewicka, Halina, Études sur l'ancienne farce française, Bibliothèque française et romane, Série A: Manuels et études linguistiques, 27 (Paris: Klincksieck; Varsovie: PWN - Éditions scientifiques de Pologne, 1974)

Liaroutzos, Chantal, Le Pays et la mémoire: Pratiques et représentations de l'espace français dans les ceuvres de Gilles Corrozet et Charles Estienne (Paris: Champion, 1998)

Lindenbaum, Peter, Changing Landscapes: Anti-Pastoral Sentiment in the English Renaissance (Athènes et Londres: University of Georgia Press, 1986)

Lovejoy, A.O., et G. Boas, Primitivism and Related Ideas in Antiquity (Baltimore: Johns Hopkins Press, 1935) 
Lumières de la Pléiade - Neuvième stage international d'études humanistes, Tours, 1965, De Pétrarque à Descartes, 11 (Paris: Vrin, 1966)

MacKenzie, Rob, «Macaronic Verse: Last Refuge of the Mongrel», (Proceedings of the Fourth Sub Voicive Colloquium, May 2000), The Gig, Ontario, 11(2002), p. $45-54$

McFarlane, I.D., A Literary History of France: Renaissance France 1470-1589 (Londres et Tonbridge: Benn; New York: Barnes and Noble, 1974)

McGowan, Margaret, Ideal Forms in the Age of Ronsard, Una's Lectures, 5 (Berkeley-Los Angeles-Londres: University of California Press, 1985)

Magnien, Michel, Étienne de la Boétie, Collection Bibliographie des Écrivains français, 7 (Paris et Rome: Memini, 1997)

Margolin, Jean-Claude, et Sylvain Matton, éds, Alchimie et philosophie à la Renaissance. Actes du colloque international de Tours (4-7 décembre 1991), De Pétrarque à Descartes, 57 (Paris: J. Vrin, 1993)

Marot, Clément, Les Fuvres poétiques de Clément Marot, éd. G. Defaux, Classiques Garnier, tome 2 (Paris: Bordas, 1993)

Martin, Rose-Ann, «Nature and the Cosmos in the Works of Remy Belleau», thèse de doctorat (PhD) sous la direction de Bodo L.O. Richter (Buffalo, New York: State University of New York at Buffalo, 1975)

Martinez, Ronald L., «Benefit of Absence: Machiavellian Valediction in Clizia», in Machiavelli and the Discourse of Literature, éd. Albert Russell Ascoli et Victoria Kahn (Ithaca et Londres: Cornell University Press, 1993), p. 117-144

Mathieu-Castellani, Gisèle, «L'Inscription-épitaphe ou le tombeau figuré», dans Dominique Moncond'Huy, Le Tombeau poétique en France (Poitiers: La Licorne, UFR Langues Littératures Poitiers, 1994), p. 143-154

Mauss, Marcel, Essai sur le don: Forme et raison de l'échange dans les sociétés archaïques, in Sociologie et anthropologie (Paris: PUF, 1950), p. 143-279

Mazouer, Charles, «Théâtre et religion dans la seconde moitié du XVIe siècle (1550-1610)», French Studies, 60 (2006), 295-304 
- «La Figure de David dans les tragédies de la Renaissance», in Claude Le Jeune et son temps en France et dans les États de Savoie, 1530-1600: musique, littérature et histoire: actes du colloque de Chambéry (4-7 novembre 1991), textes réunis par Marie-Thérèse Bouquet-Boyer et Pierre Bonniffet (Chambéry: Institut de recherche et d'histoire musicale des États de Savoie; Berne, Berlin etc.: Peter Lang, 1996), p. 253-263

- Le Personnage du naïf dans le théâtre comique du Moyen Âge à Marivaux (Paris: Klincksieck, 1979)

Mazzio, Carla, et Bradin Cormack, Book Use, Book Theory: 1500 - 1700 (Chicago: University of Chicago Press, 2005)

Mecking, Volker, analyses philologiques de l'œuvre de Belleau, sans titre, Zeitschrift für romanische Philologie, 122 (2006), p. 138-146 et p. 568-575

Mély, F. de, Lapidaires de l'antiquité et du moyen âge, vol. 1 (Paris: Ern. Leroux, 1896)

- «Les Reliques du lait de la Vierge et la galactite», Revue archéologique, $3^{\text {e }}$ série, tome 15 (1890)

Ménager, Daniel, La Renaissance et le détachement, Études et essais sur la Renaissance, 91 (Paris: Classiques Garnier, 2011)

- «Ronsard, Belleau et les blasons de 1554-1556», Nouvelle revue du seizième siècle, 14 (1996), 29-41

- La Renaissance et le rire, Perspectives littéraires (Paris: PUF, 1995)

Merrill, Robert Valentine, et Robert J. Clements, Platonism in French Renaissance Poetry (New York: New York University Press, 1957)

Millet, Ollivier de Millet et Philippe de Robert, «David et Batsheba dans la littérature française: Sens spirituel et littérature d'imagination», in W. Dietrich et H. Herkommer (éd.), König David - biblische Schlüsselfigur und europäische Leitgestalt, 19. Colloquium der Schweizerischen Akademie der Geistes- und Sozialwissenschaften (Freiburg/Schweiz: Walter Dietrich, 2003), p. 777-793

Milroy, Lesley et Pieter Muysken (éd.), One Speaker, Two Languages: CrossDisciplinary Perspectives on Code-Switching (Cambridge: Cambridge University Press, 1995) 
Moncond'Huy, Dominique, Le Tombeau poétique en France (Poitiers: La Licorne, UFR Langues Littératures Poitiers, 1994)

Monga, Luigi, Le Genre pastoral au XVle siècle: Sannazar et Belleau, Encyclopédie universitaire (Paris: Éditions Universitaires, 1974)

Moss, Ann, Printed Commonplace-Books and the Structuring of Renaissance Thought (Oxford: Clarendon Press, 1996); traduit en français sous le titre Les Recueils de lieux communs: méthode pour apprendre à penser à la Renaissance, par Patricia Eichel Lojkine, Monique Lojkine Morelec, Marie Christine Munoz Teulié et Georges Louis Tin, sous la direction de Patricia Eichel Lojkine (Genève et Paris: Droz, 2002)

Müller, Rainer A., Edelsteinmedizin im Mittelalter: Die Entwicklung der spãtantiken und mittelalterlichen Lithotherapie unter besonderer Berücksichtigung des Konrad von Megenberg (Munich: Demeter, 1984)

Musso, Jean-Michel, Aline Lecœur, Magali Kerdiles, Le Château du Grand Jardin, Joinville (Langres: Guéniot, 1993)

Naïs, Hélène, Les Animaux dans la poésie française de la Renaissance: Science Symbolique - Poésie (Paris: Didier, 1961)

Nash, Jerry C., «Per angusta ad augusta: Ronsard and the Renaissance Belief in Poetry as Therapy», in Colette H. Winn (éd.), Ronsard: figure de la variété. En mémoire d'Isidore Silver, Travaux d'humanisme et Renaissance, 368, Études Ronsardiennes, 8 (Genève: Droz, 2002), p. 53-63

- The Love Aesthetics of Maurice Scève: Poetry and Struggle (Cambridge-New York-Port Chester-Melbourne-Sydney: Cambridge University Press, 1991)

Nevile, Jennifer, «Dance and the Garden: Moving and Static Choreography in Renaissance Europe», Renaissance Quarterly, 52 (1999), p. 805-836

Nilles, Camilla J., «Imitation and Sublimation in Rémy Belleau's 'La Cerise'», Symposium, 41 (1987), 67-80

Nora, Pierre, éd., Les Lieux de mémoire, Bibliothèque illustrée des histoires, 7 vols (Paris: Gallimard, 1984-1992)

North, Helen, Sophrosyne: Self-knowledge and Self-restraint in Greek Literature, Cornell Studies in Classical Philology, 35 (Ithaca, N.Y.: Cornell University Press, 1966) 
Nutton, Vivian, «Greek Science in the Sixteenth-Century Renaissance», in J.V. Field et Frank A.J.L. James, éd, Renaissance and Revolution: Humanists, Scholars, Craftsmen and Natural Philosophers in Early Modern Europe (Cambridge: Cambridge University Press, 1993), p. 15-28

O'Brien, John, «Anacreon redivivus»: A Study of Anacreontic Translation in MidSixteenth-Century France, Recentiores: Later Latin Texts and Contexts (Ann Arbor: University of Michigan Press, 1995)

- «Ronsard, Belleau and Renvoisy», Early Music History, 13 (1994), p. 199-215

- «Theatrum catopticum: Ronsard's Amours de Cassandre», The Modern Language Review, 86 (1991), 298-309

Ong, Walter J., Orality and Literacy: The Technologizing of the Word (Londres: Methuen, 1982)

Pannier, Léopold, Les Lapidaires français du Moyen Âge des XIIe, XIIle et XIVe siècles, Fascicule 52 de la Bibliothèque de l'École des Hautes Études (Paris: Vieweg, 1882)

Pantin, Isabelle, La Poésie du ciel en France dans la seconde moitié du seizième siècle (Genève: Droz, 1995)

Pastoureau, Michel, Bleu, histoire d'une couleur (Paris: Seuil, 2000)

Peschel, Enid Rhodes, Medicine and Literature (New York: Neale Watson Academic Publications, 1980)

Picherit, Jean-Louis G., La Métaphore pathologique et thérapeutique à la fin du Moyen Âge (Tübingen: Max Niemeyer, 1994)

Pollidori, Ornella Castellani, Niccolò Machiavelli e il «Dialogo intorno alla nostra lingua» (Florence: Olschki, 1978)

Pommerol, Madame Michel Jullien de, née Marie-Henriette de Montety, Albert de Gondi maréchal de Retz, Travaux d'humanisme et Renaissance, 5 (Genève: Droz, 1953)

Pot, Olivier, Inspiration et mélancolie: L'Épistémologie poétique dans les Amours de Ronsard, Études ronsardiennes, 4; Travaux d'humanisme et Renaissance, 240 (Genève: Droz, 1990) 
Prévot, Georges, «Les Emprunts de Rémy Belleau à Jean Second dans ses Baisers ( $2^{\mathrm{e}}$ Journée de la Bergerie)», Revue d'Histoire littéraire de la France, 28 (1921), 321-339

Radcliff-Umstead, Douglas, The Birth of Modern Comedy in Renaissance Italy (Chicago et Londres: University of Chicago Press, 1969; réimpression Ann Arbor, UMI, 2000)

Raymond, Marcel, Baroque et Renaissance poétique (Paris: Corti, 1955)

Reinhardstoettner, Karl von, Plautus: Spätere Bearbeitungen plautinischer Lustspiele (Leipzig: Wilhelm Friedrich, 1886)

Reuben, Catherine, "Clément Marot's Translation of the Psalms in the Service of Reformation», in Renaissance Reflections: Essays in Memory of C.A. Mayer, éd. Pauline M. Smith et Trevor Peach (Paris: Champion, 2002), p. 107-127

Ricœur, Paul, Temps et récit, I (Paris: Seuil, 1983)

Riddle, John M., Marbode of Rennes" (1035-1123) "De lapidibus» considered as a medical treatise with text, commentary and C.W. King's translation together with text and translation of Marbode's minor works on stones (Wiesbaden: Franz Steiner, 1977)

Rigal, Eugène, Le Théâtre français avant la période classique (fin du XVle et commencement du XVIIe siècle) (Paris: Hachette, 1901)

Rigolot, François, «Métamorphoses de l'escargot: Morale de l'expression chez Remy Belleau et Francis Ponge», in (Re)Inventing the Past: Essays in Honour of Ann Moss, éd. Gary Ferguson et Catherine Hampton, Durham Modern Languages Series (Durham: University of Durham, 2003), p. 195-214

- «Poétiques de l'Huître: Francis Ponge correcteur de Remy Belleau», in Poétiques de l'Objet, éd. François Rouget et John Stout (Paris: Champion, 2001), p. 231-245

- Louise Labé Lyonnaise, ou la Renaissance au féminin (Paris: Champion, 1997)

- «Louise Labé et la redécouverte de Sappho», Nouvelle Revue du seizième siècle, 1 (1983), 19-31 
Roger-Vasselin, Bruno, Montaigne et l'art de sourire à la Renaissance (SaintGenouph: Nizet, 2003)

Rose, Steven, The Making of Memory: From Molecules to Mind (Toronto-New YorkLondres-Sydney-Auckland: Bantam Books, 1993), traduit par Marcel Blanc, La Mémoire: Des Molécules à l'esprit (Paris: Seuil, 1994)

Rosenberg, Aubrey, «A Reading of Belleau's "La Cornaline"», Romance Notes, 16 (1974-1975), p. 410-414

Rossi, Paolo, Clavis universalis: Arti della memoria e logica combinatoria da Lullo a Leibniz (Bologne: II mulino, 1983)

- Clavis universalis: Arti mnemoniche e logica combinatoria da Lullo a Leibniz (Milan: R. Ricciardi, 1960)

Rossi, Paolo L., «Society, Culture and the Dissemination of Learning» in Science, Culture and Popular Belief in Renaissance Europe, éd. Stephen Pumfrey, Paolo L. Rossi et Maurice Slawinski (Manchester: Manchester University Press, 1991), p. 143-175

Rouget, François, L'Apothéose d'Orphée: L'Esthétique de l'ode en France au XV/e siècle de Sebillet à Scaliger (1548-1561), Travaux d'humanisme et Renaissance, 287 (Genève: Droz, 1994)

Rouget, François et Colette Winn, L'Album de vers de Catherine de Clermont, Maréchale de Retz (Paris: Champion, 2004)

Ruh, Kurt, Höfische Epik des deutschen Mittelalters; 1. Von den Anfängen bis zu Hartmann von Aue, Grundlagen der Germanistik, 7 (Berlin: Erich Schmidt, 1977; première édition Berlin: Erich Schmidt, 1967)

Salmon, J.H.M., Society in Crisis: France in the Sixteenth Century (Londres et Tonbridge: Benn, 1980; première impression 1975)

Sankovitch, Tilde, «Folly and Society in the Comic Theatre of the Pléiade», in Folie et déraison à la Renaissance, Colloque international tenu en novembre 1973 sous les auspices de la Fédération Internationale des Instituts et Sociétés pour l'Étude de la Renaissance; Travaux de l'Institut pour l'Étude de la Renaissance et de l'Humanisme, 5 (Bruxelles: Université de Bruxelles, 1976), p. 99-108

Sealy, R.J. (S.J.), The Palace Academy of Henry III (Genève: Droz, 1981) 
Scarry, Elaine, The Body in Pain: The Making and Unmaking of the World (New York et Oxford: Oxford University Press, 1985)

Schama, Simon, Landscape and Memory (London: HarperCollins, 1995)

Schmidt, Albert-Marie, «Haute science et poésie française au XVle siècle» dans Études sur le XVle siècle (Paris: Albin Michel, 1967), p. 125-171

- La Poésie scientifique en France au seizième siècle: Peletier, Ronsard, Scève, Baïf, Belleau, Du Bartas, les cosmologues, les hermétistes (Paris: Albin Michel, 1938; Lausanne: Rencontre, 1970)

Schneegans, H., Geschichte der grotesken Satire (Strasbourg: Trübner, 1894)

Schreiner, Susan E., Where Shall Wisdom be Found? Calvin's Exegesis of Job from Medieval and Modern Perspectives (Chicago: University of Chicago Press, 1994)

Shaffer, E., The Garden of Health: An Account of Two Herbals, the «Gart der Gesundheit» and the «Hortus sanitatis» ([San Francisco:] Book Club of California, 1957)

Simonin, Michel, «'Poësie est un pré', 'Poëme est une fleur': métaphore horticole et imaginaire du texte à la Renaissance», dans La Letteratura e $i$ giardini. Atti del Convegno Internazionale di Studi di Verona-Garda, 2-5 Ottobre 1985. Biblioteca dell' «Archivum Romanicum», Série 1, vol. 207 (Florence: Olschki, 1987), p. 45-56

Skenazi, Cynthia, «L'Économie du don et le mécénat: Les Formes de l'échange dans une épître de Clément Marot», French Studies 57 (2003), 463-474

- Le Poète architecte en France: constructions d'un imaginaire monarchique (Paris: Champion, 2003)

Slater, Niall W., Plautus in Performance: The Theater of the Mind (Princeton, N.J.: Princeton University Press, 1985)

Smith, Gary, et Hinderk M. Emrich (éd.), Vom Nutzen des Vergessens (Berlin: Akademie Verlag, 1996)

Solomon, Michael, Fictions of Well-Being: Sickly Readers and Vernacular Medical Writing in Late Medieval and Early Modern Spain (Philadelphie et Oxford: Penn Press, 2010) 
Sontag, Susan, Regarding the Pain of Others (New York: Farrar, Straus \& Giroux, 2003; Londres: Penguin Books, 2004)

- Illness as Metaphor (New York: Farrar, Straus \& Giroux, 1978)

Starobinski, Jean, «L’Encre de la mélancolie», La Nouvelle Revue Française, 123 (1963), 410-423

Stçlen, M., "Codeswitching for Humour and Ethnic Identity: Written DanishAmerican Occasional Songs», in Codeswitching, éd. Carol M. Eastman, Multilingual Matters, 89 (Clevedon-Philadelphie-Adelaïde: Multilingual Matters, 1992), p. 215-228

Stone, Howard, «The French Language in Renaissance Medicine», Bibliothèque d'humanisme et Renaissance, 15 (1953), 315-346

Supple, James J., Arms Versus Letters: The Military and Literary Ideals in the «Essais» of Montaigne (Oxford: Clarendon Press; New York: Oxford University Press, 1984)

Sydow, Paul, Die französische Originalkomödie des XVI. Jahrhunderts, InauguralDissertation zur Erlangung der philosophischen Doktorwürde der hohen philosophischen Fakultät der Vereinigten Friedrichs-Universität HalleWittenberg (Halle a. S.: Heinrich John, 1908)

Tadié, Jean-Yves et Marc, Le Sens de la mémoire, NRF (Paris: Gallimard, 1999)

Tarugi, Luisa Rotondi Secchi (éd.), Rapporti e scambi tra umanesimo italiano ed umanesimo europeo: «L'Europa e' uno stato d'animo», Caleidoscopio, 10 (Milan: Nuovi Orizzonti, 2001)

Tetel, «La Poétique de la réflexivité chez Belleau», Studi francesi, 29 (1985), 1-18

Thorndike, Lynn, A History of Magic and Experimental Science, vols V et VI: The Sixteenth Century (New York: Columbia University Press, 1941)

Timm, L.A., «Code-Switching in War and Peace», in Aspects of Bilingualism, éd. Michel Paradis (Columbia, SC: Hornbeam, 1978), p. 302-315

Tomarken, Annette H., The Smile of Truth: The French Satirical Eulogy and Its Antecedents (Princeton: Princeton University Press, 1990) 
Tricotel, Édouard, «Vers inédits de Remy Belleau», Bulletin du Bibliophile et du Bibliothécaire, 40 (1873), 281-293

Trinkaus, Charles, In Our Image and Likeness: Humanity and Divinity in Italian Humanist Thought, 2 tomes (Londres: Constable et Chicago: University of Chicago Press, 1970; réimpr. Notre Dame, IN: University of Notre Dame Press, 1995)

Trippe, Rosemary, «The Hypnerotomachia Poliphili, Image, Text, and Vernacular Poetics», Renaissance Quarterly, 55 (2002), p. 1222-1258

Usher, Phillip John, «La Poésie sans frontières: Pour une lecture globale de Ronsard et D'Aubigné», Revue des Amis de Ronsard, 25 (2012), 103-119

Verdier, Maurice F., «Rémy BELLEAU (1528-1577), étude historique et littéraire», thèse soutenue en mai 1991, Paris IV, Sorbonne. Reproduite sur microfiches, Lille-Thèses. Doctorat Nouveau régime 91/PA04/021; publiée Lille: A.N.R.T., 1992

Viallon-Schoneveld, Marie (éd.), Médecine et médecins au XVle siècle: Actes du neuvième colloque du Puy-en-Velay (Saint-Étienne: Presses Universitaires de Saint-Étienne, 2002)

Vignes, Jean, «Paraphrase et appropriation: Les avatars poétiques de l'Ecclésiaste au temps des Guerres de Religion (Dalbiac, Carle, Belleau, Baïf)», Bibliothèque d'humanisme et Renaissance, 55 (1993), 503-526

Wagner, Hermann, Remy Belleau und seine Werke. Inaugural-Dissertation der hohen philosophischen Fakultät der Universität Leipzig zur Erlangung der Doktorwürde (Leipzig-Reudnitz: Oswald Schmidt, 1890)

Watson, George, Writing a Thesis: A Guide to Long Essays and Dissertations (Londres et New York: Longman, 1990; publié pour la première fois en 1987)

Weber, Henri, La Création poétique au XVle siècle en France de Maurice Scève à Agrippa d'Aubigné (Paris: Nizet, 1955, réimpr. 1981)

Weijers, Olga et M. Gumbert-Hepp (éd.), Lexicon Latinitatis Nederlandicae Medii Aevi (Leiden: Brill, 1986)

Wenzel, Siegfried, Macaronic Sermons: Bilingualism and Preaching in LateMedieval England (Ann Arbor: The University of Michigan Press, 1994) 
Whitfield, J.H., «On Machiavelli's Use of 'Ordini'», Italian Studies, 10 (1955), p. 19-39; réimprimé dans ses Discourses on Machiavelli (Cambridge: Heffer, 1969), p. $141-162$

Wickersheimer, Charles, La Médecine et les médecins en France à l'époque de la Renaissance (Paris: A. Maloine, 1905; Genève: Slatkine, 1970)

Wilson, Dudley B., French Renaissance Scientific Poetry (Londres: Athlone, 1974)

- Descriptive Poetry in France from Blason to Baroque (Manchester: Manchester University Press; New York: Barnes and Noble, 1967)

Wind, Edgar, Pagan Mysteries in the Renaissance (Harmondsworth: Penguin, 1967)

Windfuhr, Manfred, Die barocke Bildlichkeit und ihre Kritiker: Stilhaltungen in der deutschen Literatur des 17. und 18. Jahrhunderts (Stuttgart: Metzler, 1966)

Wittschier, Heinz Willi, Die Lyrik der Pléiade (Frankfurt-am-Main: Athenäum, 1971)

Woolard, K.A., «Codeswitching and Comedy in Catalonia», dans Codeswitching, éd. Monica Heller (q.v.), p. 53-76

Yates, Frances A., The Art of Memory (Londres, Melbourne et Henley: Ark Paperbacks, 1984; première édition 1966); traduction par Daniel Arasse, L'Art de la mémoire (Paris: Gallimard, 1975)

- The French Academies of the Sixteenth Century (Londres: Warburg Institute, 1947). Traduction de L'anglais, Les Académies en France au XVle siècle, Th. Chaucheyras, Questions (Paris: PUF, 1996)

Zambelli, Paola, «Le Problème de la magie naturelle à la Renaissance», in Magia, astrologia e religione nel Rinascimento, Convegno polacco-italiano (Varsavia: 25-27 settembre 1972), Conferenze, Fascicolo 65 (Wrocław, Varsovie, Kraków, Gdańsk: Ossolineum, 1974 (p. 75-76)

Zinguer, Ilana, et Isabelle Martin, éd., Théâtre de l'anatomie et corps en spectacle: Fondements d'une science de la Renaissance, actes du colloque Les Théâtres de l'anatomie Haïfa, 17-19 décembre 2002 (Berne: Peter Lang, 2006) 


\section{Manuscrit}

MS Nouv. Acq. Fr. 4021 à la BnF, «Histoire chronologique et topographique des Princes et Seigneurs de la Ville de Joinville»

\section{Sites web}

http://armae.it/moderne/132cuirasses.htm, consulté le 20 mai 2011

http://www.finns-books.com/flowersf.htm, consulté le 7 juillet 2011

http://www.levity.com/alchemy/bloomfld.html, consulté le 20 juillet 2011

www.psychoanalyse-salzburg.com/sap_zeitung/pdf/Kirchner.pdf, consulté le 26 avril 2013 